OPEN ACCESS

Edited by:

Jianbo Xiao,

University of Macau, Macau

Reviewed by:

Weibin Bai,

Jinan University, China

Washim Khan,

Jamia Hamdard, India

*Correspondence:

Hui Dong

tjdonghui@163.com

Fuer Lu

felu@tjh.tjmu.edu.cn

tThese authors have contributed equally to this study.

Specialty section: This article was submitted to

Ethnopharmacology,

a section of the journal

Frontiers in Pharmacology

Received: 22 October 2016 Accepted: 19 January 2017

Published: 03 February 2017

Citation: Gong J, Hu M, Huang Z, Fang K,

Wang $D$, Chen $Q, L i$ J, Yang $D$,

Zou $X, X u$ L, Wang $K$, Dong $H$ and

Lu $F$ (2017) Berberine Attenuates Intestinal Mucosal Barrier Dysfunction

in Type 2 Diabetic Rats.

Front. Pharmacol. 8:42.

doi: 10.3389/fphar.2017.00042

\section{Berberine Attenuates Intestinal Mucosal Barrier Dysfunction in Type 2 Diabetic Rats}

\author{
Jing Gong ${ }^{1+}$, Meilin Hu ${ }^{1+}$, Zhaoyi Huang ${ }^{2}$, Ke Fang', Dingkun Wang ${ }^{2}$, Qingjie Chen ${ }^{3}$, \\ Jingbin $\mathrm{Li}^{2}$, Desen Yang ${ }^{1,4}$, Xin Zou ${ }^{1}$, Lijun Xu ${ }^{1}$, Kaifu Wang ${ }^{1}$, Hui Dong ${ }^{1 *}$ and Fuer Lu ${ }^{1 *}$ \\ 1 Institute of Integrated Traditional Chinese and Western Medicine, Tongji Hospital, Tongji Medical College, Huazhong \\ University of Science and Technology, Wuhan, China, ${ }^{2}$ Department of Integrated Traditional Chinese and Western Medicine, \\ Tongji Hospital, Tongji Medical College, Huazhong University of Science and Technology, Wuhan, China, ${ }^{3}$ Department of \\ Biochemistry and Molecular Biology, Tongji Medical College, Huazhong University of Science and Technology, Wuhan, \\ China, ${ }^{4}$ Department of Pharmacy, Hubei University of Traditional Chinese Medicine, Wuhan, China
}

Background: Intestinal mucosal barrier dysfunction plays an important role in the development of diabetes mellitus (DM). Berberine (BBR), a kind of isoquinoline alkaloid, is widely known to be effective for both DM and diarrhea. Here, we explored whether the anti-diabetic effect of BBR was related to the intestine mucosal barrier.

Methods and Results: The rat model of T2DM was established by high glucose and fat diet feeding and intravenous injection of streptozocin. Then, those diabetic rats were treated with BBR at different concentrations for 9 weeks. The results showed, in addition to hyperglycemia and hyperlipidemia, diabetic rats were also characterized by proinflammatory intestinal changes, altered gut-derived hormones, and 2.77-fold increase in intestinal permeability. However, the treatment with BBR significantly reversed the above changes in diabetic rats, presenting as the improvement of the high glucose and triglyceride levels, the relief of the inflammatory changes of intestinal immune system, and the attenuation of the intestinal barrier damage. BBR treatment at a high concentration also decreased the intestinal permeability by $27.5 \%$ in diabetic rats. Furthermore, BBR regulated the expressions of the molecules involved in TLR4/MyD88/NF-kB signaling pathways in intestinal tissue of diabetic rats.

Conclusion: The hypoglycemic effects of BBR might be related to the improvement in gut-derived hormones and the attenuation of intestinal mucosal mechanic and immune barrier damages.

\section{Keywords: berberine, diabetes, intestinal mucosal barrier, gut immune system, gut-derived hormones}

\section{INTRODUCTION}

The number of diabetics underwent a four-fold change over the past 35 years, and worldwide incidence of diabetes mellitus (DM) was about 9\% in 2014 (Lancet, 2016a). In China, the incidence was $11.6 \%$, and the rate of prediabetes was $50.1 \%$ among adults. However, only $25.8 \%$ of diabetics accepted hypoglycemic treatment, and only $39.7 \%$ population of the treated diabetic patients

Abbreviations: BBR, berberine; GIP, gastric inhibitory polypeptide; GLP-1, glucagon-like peptide-1; HGFD, high glucose and fat diet; LP, lamina propria; LPS, lipopolysaccharide; MLN, mesenteric lymph nodes; OCLN, occludin-1; PPRs, pattern recognition receptors; STZ, streptozotocin; T2DM, Type 2 diabetes. 
achieved eligible glucose control (Xu et al., 2013). In 2012, it was reported that poor blood glucose control led to the deaths of 370 million diabetics (Lancet, 2016b). High mortality rate of diabetes brings great health burden to the world populations, arousing researchers to seek out new therapies of diabetes based on new insight into the pathogenesis. Among these, the role of intestine has attracted great concerns.

The intestinal immune system is the first defense line for the contact with dietary antigens. Intestinal changes caused by intestinal infection, oral milk or gluten protein antigens, and varied intestinal flora has been confirmed involved in the pathogenesis of DM (Luck et al., 2015). Prior to the occurrence of diabetes, the intestinal morphology and gut immune system have changed, and intestinal permeability increases (Graham et al., 2004). On the other hand, mesenteric lymphocytes transplantation of non-obese diabetic mice could transfer diabetes to the recipient mice, which indicates DM-caused $\mathrm{T}$ cells exist in the gut immune tissue (Antvorskov et al., 2014). As is well known, T2DM is a chronic inflammatory disease; many factors are associated with the onset of T2DM including intestinal flora disturbance, immune tolerance deficiency, intestinal barrier damage, pattern recognition receptor (PPRs) expressions changes in intestinal epithelial and immune cells and gut hormone change (Winer et al., 2016). With intestinal barrier defect, LPS and other intestinal bacteria products filter into the circulation and result in local and systemic chronic inflammation, which makes islet beta cell dysfunction and insulin resistance (Burcelin, 2012). However, the role of gut immune cells and inflammation in the pathogenesis of DM are still not fully explored.

Berberine (BBR, [C20H18NO4]+), a kind of isoquinoline alkaloid, could play hypoglycemic and hypolipidemic roles in clinical and experimental studies (Dong et al., 2012, 2013). Some studies illuminated diversified action mechanisms of BBR for treating diabetes. For example, BBR could increase the glucose uptake and consumption in adipose and muscle cells, elevate insulin receptor expressions of liver and muscle, and enhance liver low density lipoprotein receptor expressions to decrease serum cholesterol and glucose (Pirillo and Catapano, 2015). Moreover, Th1 and Th17 cells differentiation and macrophage trafficking could be blocked by BBR, thereby proinflammatory cytokine secretions are reduced (Pirillo and Catapano, 2015). However, BBR is extensively known as a medicine for the treatment of diarrhea in China. In the gut, BBR could stimulate the secretions of GLP-1, depress the activity of $\alpha$-glucosidase and reduce the intestinal absorption of glucose (Leng et al., 2004; Turner et al., 2008; Zhao et al., 2012).

Taken the low oral bioavailability conundrum of BBR as well as its effect on diarrhea into account (Pirillo and Catapano, 2015), we hypothesized that BBR might prevent the development of diabetes through its action on the intestines. Metformin have also been shown to alter the intestinal flora, modulate gut hormones and maintain the intestinal barrier integrity (Shin et al., 2014; DeFronzo et al., 2016; Mardinoglu et al., 2016). Therefore, we conducted a study to testify whether the hypoglycemic effects of $\mathrm{BBR}$ as well as metformin were via preventing intestinal mucosal barrier damage and stimulating gut hormones release.

\section{MATERIALS AND METHODS}

\section{Animals, Grouping and Modeling}

Male Wistar rats with weight between 160 and $180 \mathrm{~g}$ were purchased from Hua Fukang Co., Ltd and bred in SPF circumstances in the experimental animal center of Huazhong University of Science and Technology. After acclimatization for 2 weeks, the rats were randomly divided into normal group (NO group) and HGFD group (HGFD group). The rats in NO group was fed with standard laboratory rat chow (the formula contains $35 \%$ flour, $20 \%$ corn meal, $20 \%$ soy meal, $15.5 \%$ bran, $5 \%$ fish meal, $1 \%$ dusty yeast, $0.5 \%$ bean oil, $2.5 \%$ bone meal, and $0.5 \%$ salt) while the diet in HGFD group contained $67.5 \%$ standard laboratory rat chow, $12 \%$ lard, $2 \%$ cholesterol, $0.5 \%$ bile salts and $20 \%$ sugar. The dietary regimens of the rats in different groups were unchanged until the end of the experiment. One month later, intravenous injection of small dose streptozotocin (STZ, 24mg/kg) was conducted in the rats of HGFD groups (Duca et al., 2015). Then the normal 95\% confidence intervals at various time points in OGTT were achieved according to the blood glucose of rats in NO group. If the blood glucose was greater than $20 \%$ of the upper limit of confidence interval at any time point, the rats were chosen for further studies as successful models of diabetic rats. Diabetic rats were further randomly divided into model group (MO group) and treatment groups, with 12 rats in each group. All experiments were approved by the animal ethics committee of Huazhong University of Science and Technology (NO.2015S508).

\section{Preparation of BBR and Treatment Methods}

The treatment groups included BBR treatment groups with BBR at high (BH group), middle (BM group), or low dose (BL group) and metformin group (ME group). BBR was purchased from Yabang Pharmaceutical Co. Ltd. in China (H20053180) and dissolved with $0.5 \%$ carboxymethyl cellulose sodium glue. High, medium and low dosages in BBR treatment groups were 375, 187.5 and $93.75 \mathrm{mg} \cdot \mathrm{kg}^{-1} \cdot \mathrm{day}^{-1}$ respectively (Leng et al., 2004). Daily dose of metformin was $184 \mathrm{mg} / \mathrm{kg}$ for the rats in $\mathrm{ME}$ treatment group. Daily lavage volume of each rat was $1 \mathrm{ml} / 100 \mathrm{~g}$ weight, and the solution concentration was calculated according to the lavage capacity. In addition, $0.5 \%$ sodium carboxymethyl cellulose solution was intragastric administrated to the rats in NO and MO groups. Gastric irrigation doses were adjusted weekly based on rats' weights for 9 weeks.

\section{Sampling and Metabolic Studies}

When the interventions were over, six rats in each group were used for the detections of intestinal permeability and the other six rats in each group were employed for metabolic studies and sampling. When performing OGTT, rats were overnight fasting for $12 \mathrm{~h}$ and $2 \mathrm{~g} / \mathrm{kg}$ glucose was administrated. The blood from tail veins of each rat were collected at $0,30,60$, and 120 min after the intragastric administration and the blood glucose levels were detected using a glucose monitor (Roche, German). 
At the end of 9 weeks' treatment, all experimental animals were killed. Anesthesia was performed by intraperitoneal injection with $50 \mathrm{mg} / \mathrm{kg}$ pentobarbital sodium. Abdominal aorta blood sampling, intestines and MLN were collected under sterile conditions. Samples were fixed with $4 \%$ formaldehyde or preserved in $-80^{\circ} \mathrm{C}$.

The concentration of serum insulin was detected by using iodine radioimmunoassay kit (Jinding, China). For the detection of triglyceride (TG) content, colorimetric assay (Mingdian, China) was used.

\section{Intestinal Histological Morphology Studies}

Intestine sections were stained using hematoxylin and eosin (H\&E) to assess the histological morphology. Transmission electron microscope (Hitachi, Japan) was used to evaluate the ultrastructural changes. Tissue blocks were fixed with glutaraldehyde. After rinsing and dehydration in ethanol, the tissues were embedded and sliced with ultramicrotome. Following uranyl acetate and lead citrate double staining, transmission electron microscope was used for the morphology observation and taking pictures.

\section{Intestinal Permeability Test}

FITC-dextran (Sigma, FD-4, USA) perfusion assay was used to detect intestinal epithelial permeability in the rats. After anesthesia, abdominal middle incision and ileum incision $5 \mathrm{~cm}$ distance from the appendix was performed and 10-cm-long ileal segment was ligated with 2.0 silk thread. Subsequently, $1 \mathrm{ml}$ FITC-dextran $(10 \mathrm{mg} / \mathrm{ml})$ was affused into the ligated intestinal cavity. Intestines were returned back to the abdominal cavity and kept wet by wrapping moist gauze and a plastic film. Thirty minutes later, blood was collected from the abdominal aorta. Serum was separated and fluorescence was detected with a spectrophotometer (Synergy2, USA).

\section{Flow Cytometry}

Mesenteric lymph nodes was grinded with strainer mesh and glass rods in the 1640 culture medium containing 2\% FBS. Erythrocyte was lysed and the supernatant was dismissed after centrifugation. If the markers were expressed in the cytoplasm and nucleus, FIX/PERM liquid was added to break the cell membrane. Otherwise, the cells were incubated with different antibodies for $30 \mathrm{~min}$ away from light and fixed with $1 \%$ paraformaldehyde. $\mathrm{T}$ cells were stained with CD3-FITC, CD4-APC and CD8-PE (BD Bioscience, USA); Macrophages were with CD11b-APC (BD Bioscience, USA) and CD68-FITC (AbD Serotec, UK), Tregs were with CD4-APC and Foxp3-PE (eBioscience, USA); dendritic cells (DC) were with OX62-FITC (Abcam, UK). Flow cytometry (BD FACSCalibur, USA) was applied for the detection of cells' population.

\section{Immunofluorescence Test}

Intestinal tissues were separated, rinsed, fixed and cut following paraffin embedding. After using the spontaneous fluorescence quenching agent, the sections were immunostained overnight with primary antibodies against NF- $\mathrm{B}$ (CST, USA) with triton damaging cell membranes. Then fluorescence second antibodies were co-incubated at room temperature for $30 \mathrm{~min}$. Following the rinse, the sections were stained with DAPI (Sigma, USA) for $3 \mathrm{~min}$ and sealed. Finally, observation and taking photos were performed under a Nikon fluorescence microscope.

\section{Real-Time PCR Assay}

After the extract of intestinal RNA with Trizol reagent (Takara, China) and concentration determination of RNA, reverse transcriptase kit (Takara, China) was used to synthetize the cDNA. Then, amplification was conducted using SYBR premix EX Taq $^{\mathrm{TM}}$ kit (Takara, China) in StepOne PCR detetor (Stepone, USA). Relative expressions of mRNA were calculated by $2^{-\Delta \Delta C T}$. The primer sequences are listed in Table $\mathbf{1 .}$

\section{Western Blot Assay}

Proteins of intestines (different sampling volumes for various antibodies) were separated through gel electrophoresis, transferred onto NC membranes, and incubated with antibodies against TLR4 (NOVUS, USA), Occludin-1 (OCLN, Gene Tex, USA), ZO-1 (Invitrogen, USA), Claudin-1 ( Abcam, UK), MyD88 (CST, USA), p-IKK $\beta$ (Gene Tex, USA),IKK $\beta$ (Gene Tex, USA) and $\beta$-actin (Affinity, USA). The membranes were then coincubated with fluorescent second antibodies. Oddesy imaging system was implemented to analyze the protein expressions.

\section{ELISA}

Amylin, GLP-1, GIP and Ghrelin (Ray Biotech, USA) levels in serum were tested using ELISA Kit in line with the competitive enzyme immunoassay principle. In brief, $100 \mu \mathrm{L}$ serum was added to each well and OD values were detected at $450 \mathrm{~nm}$ using a spectrophotometer (Synergy2, USA).

\section{Statistical Analysis}

Data were analyzed by SPSS 20.0 software. Statistical significance was assessed by ANOVA following K-S normality test. With homogeneity of variance, significance between different groups was evaluated by Bonferroni test; alternatively, Dunnett's T3 test was used. $P$-values $<0.05$ suggested that significant differences existed.

\begin{tabular}{lll}
\hline \multicolumn{2}{l}{ TABLE $\mathbf{1}$ | Primers for RT-PCR assay. } \\
\hline Gene & Forward $\left(\mathbf{5}^{\prime} \rightarrow \mathbf{3}^{\prime}\right)$ & \\
\hline LBP & CCCAGACGCTGGATGTGATG & TGATCTGAGATGGCAAAGTAGACC \\
CD14 & CTTATGCTCGGCTTGTGCTGT & TAGCAGCGGACACTTCCTCGT \\
IL-1 $\beta$ & GTCGGGACATAGTTACTTCAC & GACTTGGCAGAGGACTTCAC \\
TNF- $\alpha$ & CCAGGTCTCTTCAAGGGACAA & GGTATGAATGGCAAATCGGCT \\
IL-10 & GCTGGACAACATACTGCTGAC & AATGCTCCTTGATTCTGGG \\
IL-4 & CTGTCACCCTGTCTGCTTCTC & TाCTGTGACCTGGTCAAAGTGT \\
TGF- $\beta$ & AAGGACCTGGGTGGAAGT & CGGGTTGTTTGGTGTAGA \\
IL-6 & AATCTGCTCTGGTCTTCTGGA & CAGTATTGCTCTGAATGACTCTGG \\
MIF & GACTTTAGTGGCACGAGCG & GCTTGCTGTAGTTGCGGTCT \\
$\beta-$-actin & AGCCATGTACGTAGCCATCC & CTCTCAGCTGTGGTGGTGAA \\
& &
\end{tabular}


A

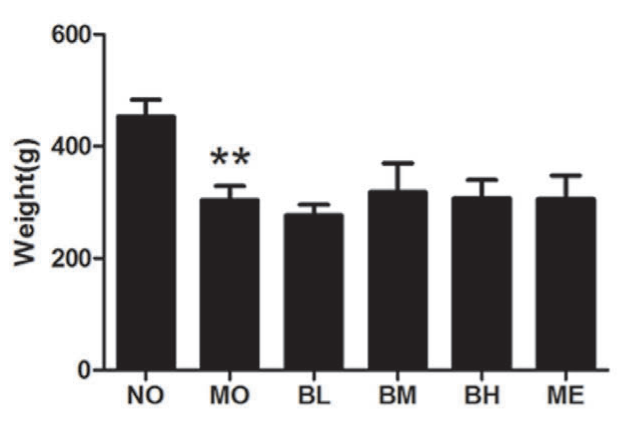

C

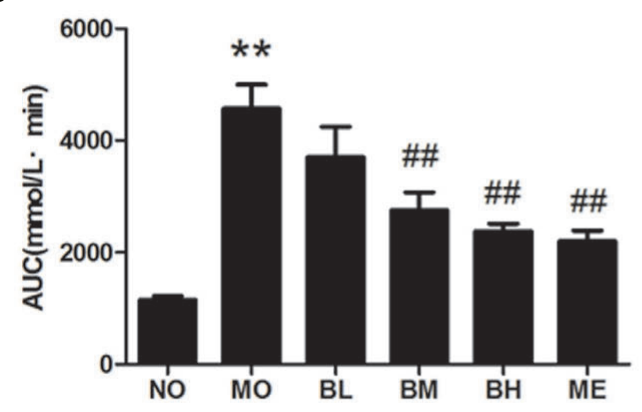

$\mathbf{E}$

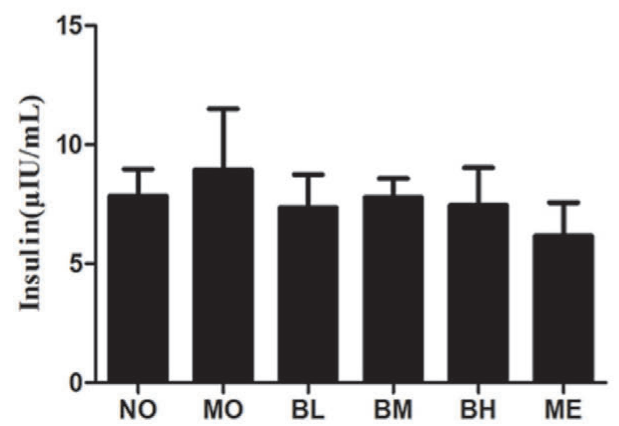

B
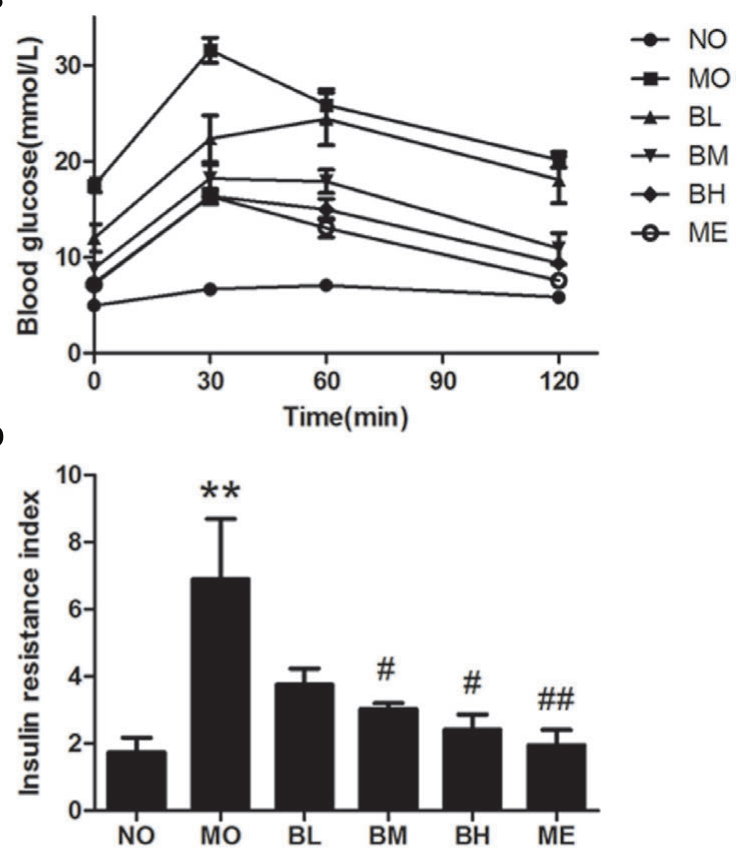

$\mathbf{F}$

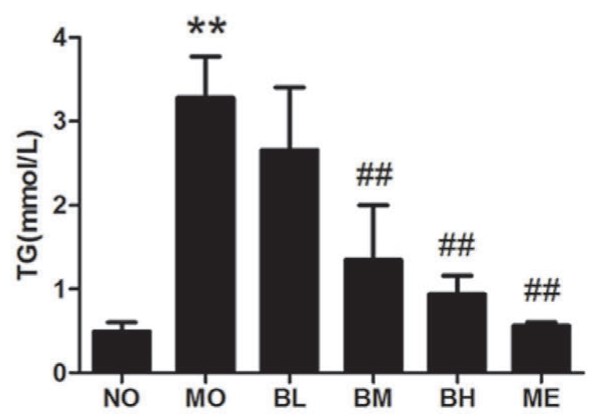

FIGURE 1 | Berberine (BBR) is effective on maintaining glucose homeostasis. (A) Body weights of rats in each group. (B) OGT of the rats after an oral gavage of $2.0 \mathrm{~g} / \mathrm{kg}$ glucose. Data presented as mean $\pm \mathrm{SE}$. $P<0.01$ for NO vs. MO group at each time point; $P<0.05$ for MO vs. BM group, MO vs. BH group and $\mathrm{MO}$ vs. ME group at each time point; $P<0.05$ for $\mathrm{MO}$ vs. BL group at 30 min after glucose administration. (C) AUC in OGTT. (D) IRI after the treatment.

(E) Serum insulin concentration of the rats. (F) Serum TG concentration. NO, normal control group; MO, diabetic model group; BL, BBR treatment group with a low concentration; BM, BBR treatment group with a middle concentration; BH, BBR treatment group with a high concentration; ME, metformin treatment group; Data presented as mean \pm SD except OGTT. $n=6$ rats in each group. ${ }^{*} P<0.01$ vs. NO group, ${ }^{\#} P<0.05$ vs. MO group, ${ }^{\# \#} P<0.01$ vs. MO group.

\section{RESULTS}

\section{BBR Attenuates the Disturbance of Glucose and Lipid Metabolism in Diabetic Rats}

Firstly, we detected the effects of BBR on glucose and lipid metabolism in diabetic rats (Figure 1). Consistent with previous study, diabetic rats underwent the weight loss after modeling (Wang et al., 2016), and BBR interventions did not significantly improve the decreased body weights. With $2 \mathrm{~g} / \mathrm{kg}$ glucose lavage in OGTT, the blood glucose at each time-point increased significantly in diabetic rats, whereas BBR at middle $(187.5 \mathrm{mg} / \mathrm{kg})$ and high dosage $(375 \mathrm{mg} / \mathrm{kg})$ reversed elevated fasting and postprandial blood glucose. The area under the concentration-time curve (AUC) in OGTT and serum TG levels also decreased in BBR-treated rats. Although no significant difference was shown with regards to serum insulin concentration in different groups, BBR decreased insulin resistance index (IRI) as well as TG in diabetic rats of BM and $\mathrm{BH}$ groups. These data indicated that BBR had specific beneficial effects on metabolic parameters. Similar hypoglycemic and lipidlowering effects were observed in ME group that metformin was administrated as a positive control.

\section{BBR Changes the Proportion of Intestinal Immune Cells in MLN of Diabetic Rats}

Flow cytometry was used to detect the proportions of $\mathrm{T}$ cells, macrophages and DC in MLN. As shown in Figure 2, the 
A
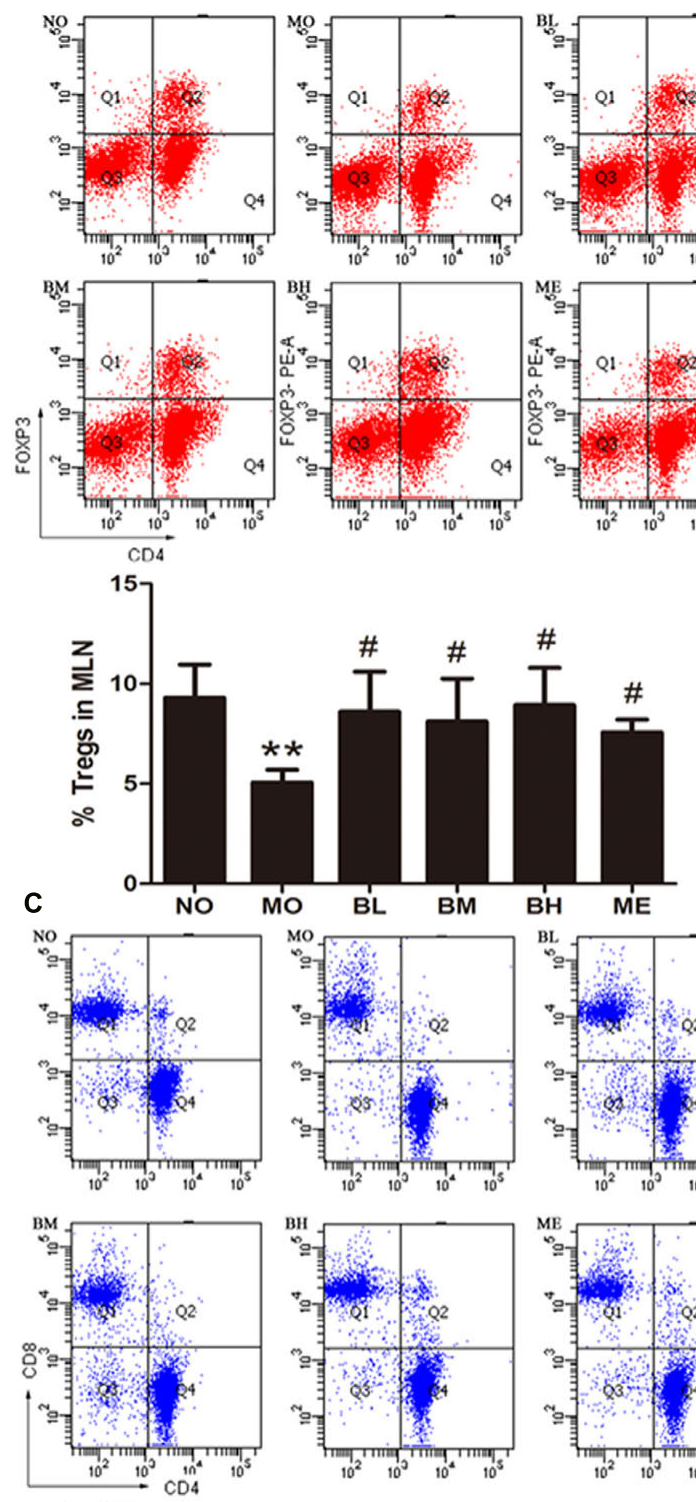

gated on $\mathrm{CD} 3+$
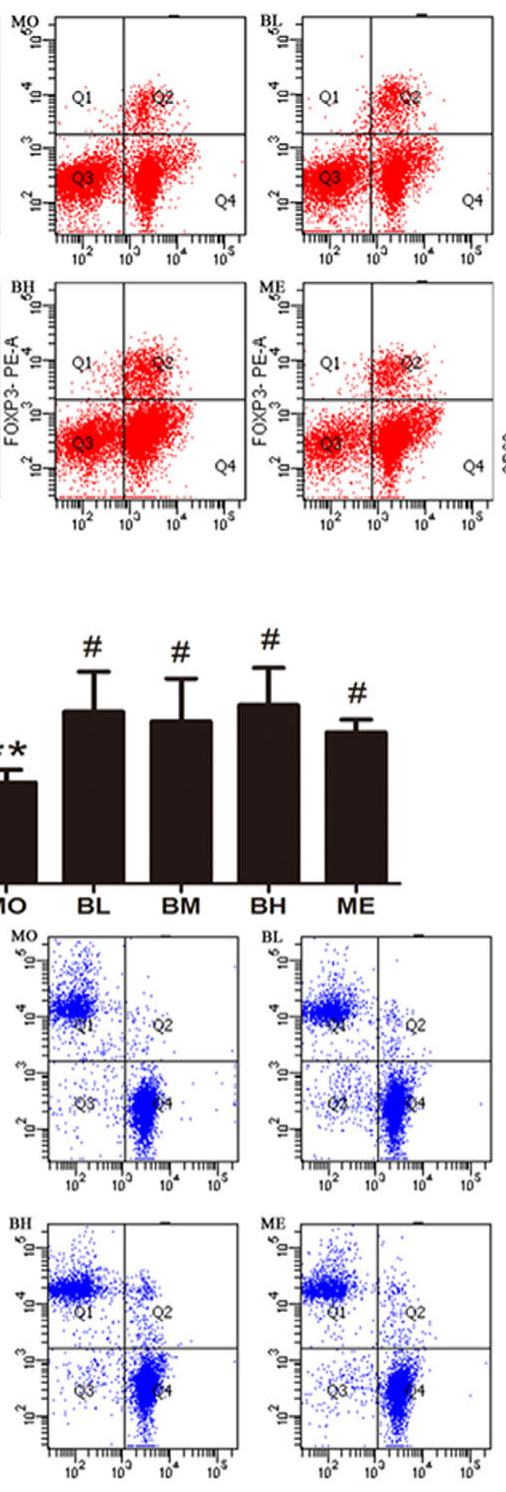

B
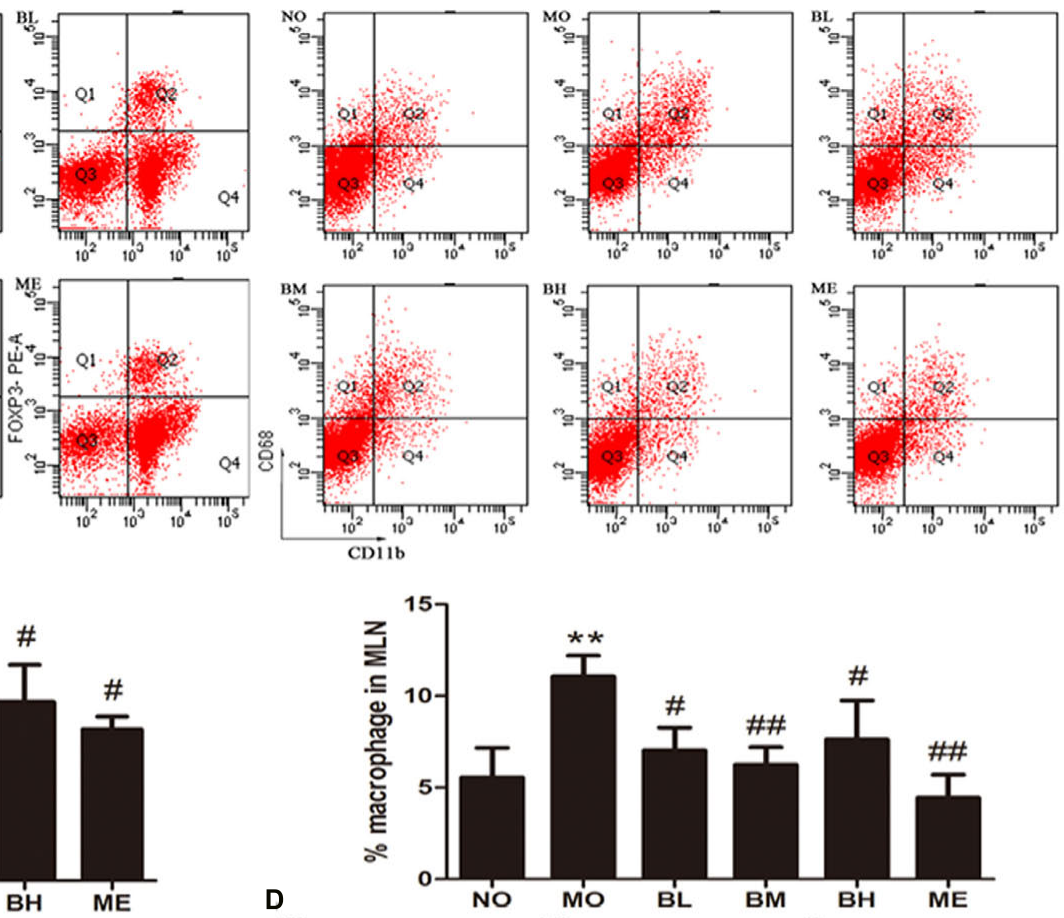

D
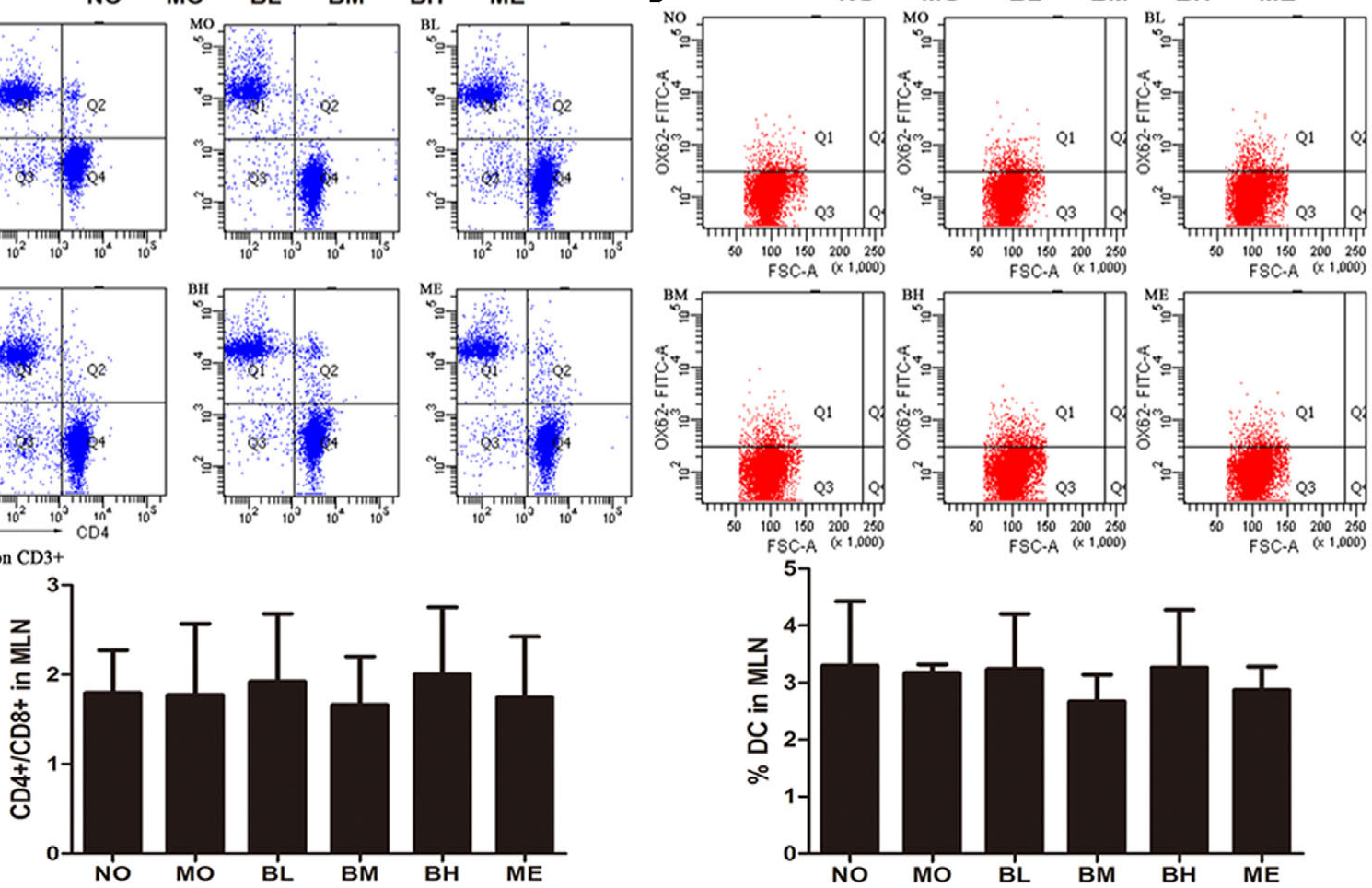

FIGURE 2 | Berberine increased Tregs and reduced macrophages percentages in MLN of diabetic rats. (A) Tregs populations in MLN ( $n=4)$.

(B) Macrophage populations of different groups in MLN $(n=4$ or 5). (C) Ratio of CD4+ and CD8+ cells on CD3+ gate ( $n=5$ or 6$)$. (D) Dendritic cell populations in $\operatorname{MLN}(n=3)$. Data presented as mean $\pm \mathrm{SD} .{ }^{* *} P<0.01$ vs. NO group, ${ }^{\#} P<0.05$ vs. MO group, ${ }^{\# \#} P<0.01$ vs. MO group.

percentage of Tregs in MLN of diabetic rats was decreased while it was increased in BBR- and metformin-treated rats. Because macrophages are the sources of many proinflammatory factors, we studied the proportion differences of macrophages with BBR administration. Significantly higher numbers of $\mathrm{CD} 11 \mathrm{~b}+\mathrm{CD} 68+\mathrm{MLN}$ cells were observed in MO group while 
A

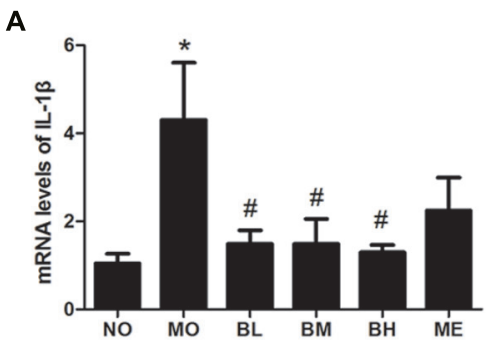

D

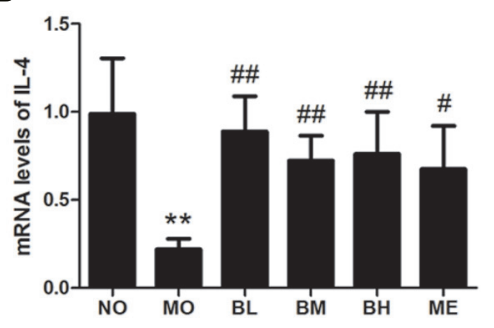

G

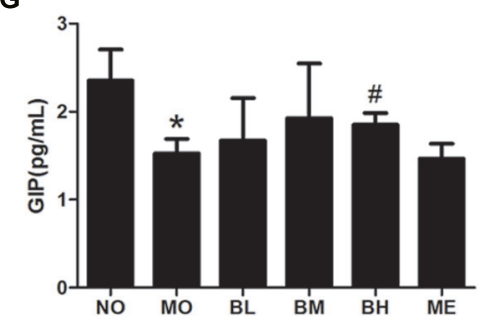

B

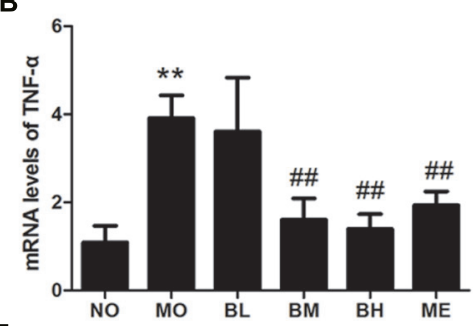

E

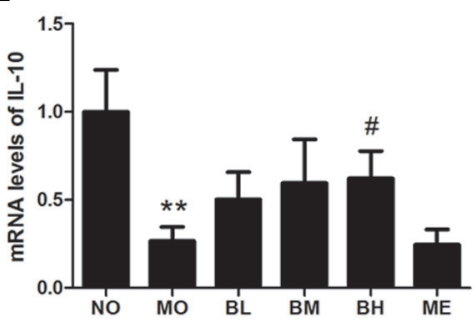

H

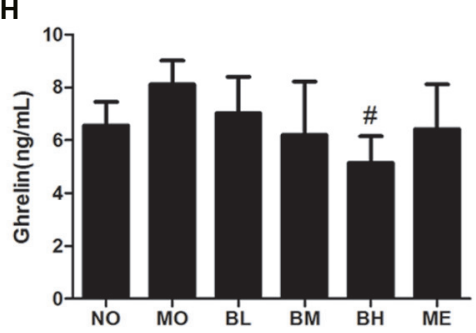

C

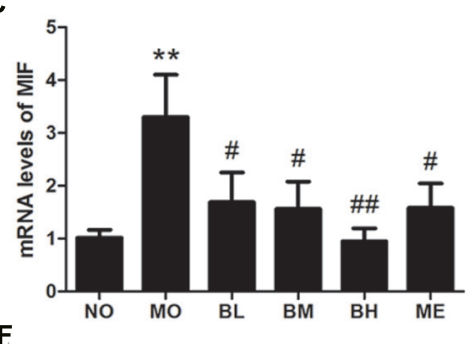

$F$

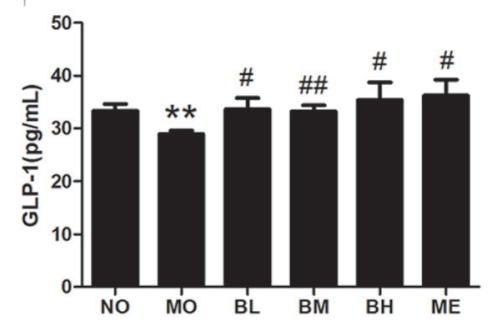

I

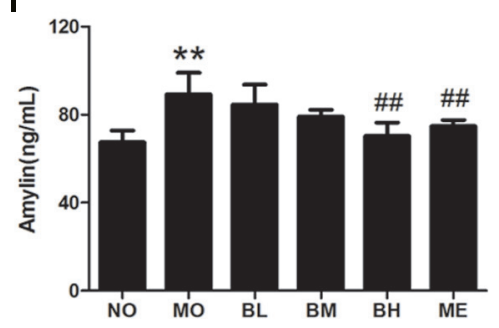

FIGURE 3 | Berberine affected mRNA expressions of intestinal cytokines and plasma gastrointestinal hormones. (A) IL-1 $\beta$ mRNA levels. (B) TNF- $\alpha$ mRNA levels. (C) MIF mRNA levels. (D) IL-4 mRNA levels. (E) IL-10 mRNA levels. (F) Plasma GLP-1 concentrations. (G) Plasma GIP concentrations. (H) Plasma Ghrelin concentrations. (I) Plasma Amylin concentrations. The levels of mRNA were expressed relative to the $\beta$-actin. Data presented as mean \pm SD. $n=6$ for each group. ${ }^{\star} P<0.05$ vs. NO group, ${ }^{\star \star} P<0.01$ vs. NO group, ${ }^{\#} P<0.05$ vs. MO group, ${ }^{\# \#} P<0.01$ vs. MO group.

less numbers were observed in BBR treatment and ME groups. As far as CD4 $+\mathrm{T}$ cells, CD8 $+\mathrm{T}$ cells, the ratio of CD4+ to CD8+ cells and DC were concerned, no significant changes were found.

\section{BBR Affects the Expressions of Intestinal Immune Factors in Diabetic Rats}

Intestinal immune factors involved in the maintenance of intestinal immune tolerance and the intestinal barrier integrity. Since BBR had the beneficial effect on immune cells of intestinal immune system, it might also influence the expressions of intestinal immune factors. The results showed that IL- $1 \beta$, TNF$\alpha$ and MIF mRNA expressions were elevated in comparison with NO group, while IL-4 and IL-10 mRNA levels were reduced in intestinal tissue of diabetic rats (Figures 3A-E). However, BBR, especially at high doses, showed significant inhibitory effects on the expressions of IL- $1 \beta$, MIF and TNF- $\alpha$ mRNA. Moreover, BBR treatment also exhibited the increase in IL-4 and IL-10 mRNA expressions in intestinal tissue of diabetic rats. Despite increased tendency for IL-6 mRNA expression and decreased trend for TGF- $\beta$ mRNA expression in MO group, heterogeneity of variance was large and no statistical difference was found in the treatment groups (Data not shown).

\section{BBR Regulates the Secretion of Gastrointestinal Hormones in Diabetic Rats}

Since gastrointestinal hormones participate in glycemic control, we next examined the effects of BBR on plasma gastrointestinal hormones levels (Figures 3F-I). After HGFD and the administration of STZ, GLP-1 and GIP secretion reduced while pancreatic beta cells-derived Amylin increased in diabetic rats (Figures 3F,I). The administration of $\mathrm{BBR}$ at different concentrations increased the secretion of GLP-1, but only BBR at a high dose influenced the production of GIP and Amylin. In the analysis regarding Ghrelin, BBR at a high dose decreased Ghrelin production compared with MO group, and no differences were found between other different groups.

\section{BBR Improves Intestinal Mucosal Barrier Function in Diabetic Rats}

High glucose and fat diet and intestinal inflammation could disrupt the intestinal mucosal barriers, leading to endotoxemia and the development of DM. Thus, we studied the effects of $\mathrm{BBR}$ on intestinal tight junction proteins expressions and mucosal barrier permeability. Tight junction protein OCLN, ZO-1 and Claudin-1 expressions were detected using western 

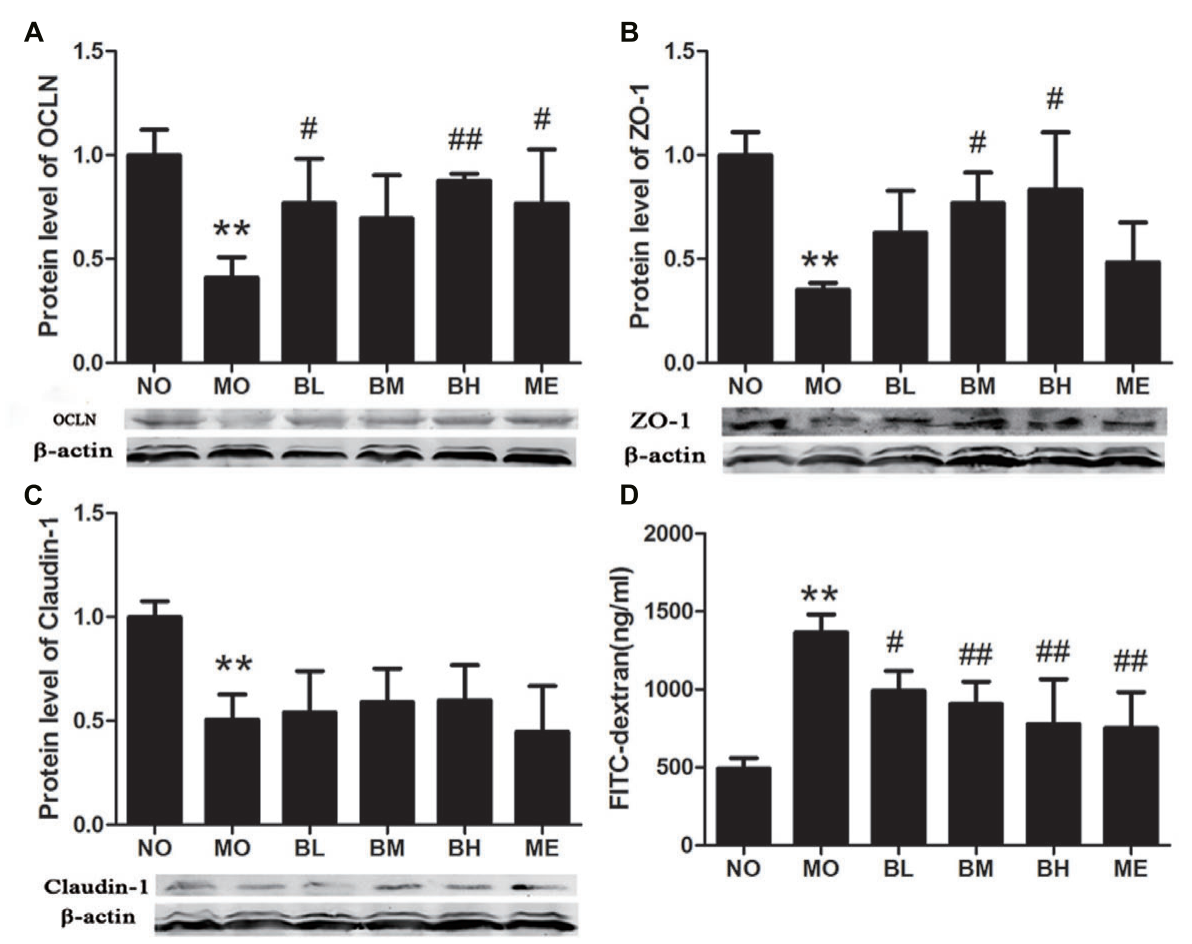

FIGURE 4 | Berberine protects intestinal mucosal integrity. (A) OCLN expression in intestine. (B) ZO-1 expression in intestine. (C) Claudin-1 expression in intestine. (D) Serum dextran concentration after the lumina infusion of FITC-dextran. The protein sampling mass was 150 or $200 \mu \mathrm{g}$. Data presented as mean $\pm \mathrm{SD}$. $n=3$ for the detections of OCLN, ZO-1 and Claudin- 1 expressions; $n=6$ for serum dextran concentration. ${ }^{* *} P<0.01$ vs. NO group, ${ }^{\#} P<0.05$ vs. MO group, ${ }^{\# \#} P<0.01$ vs. MO group.

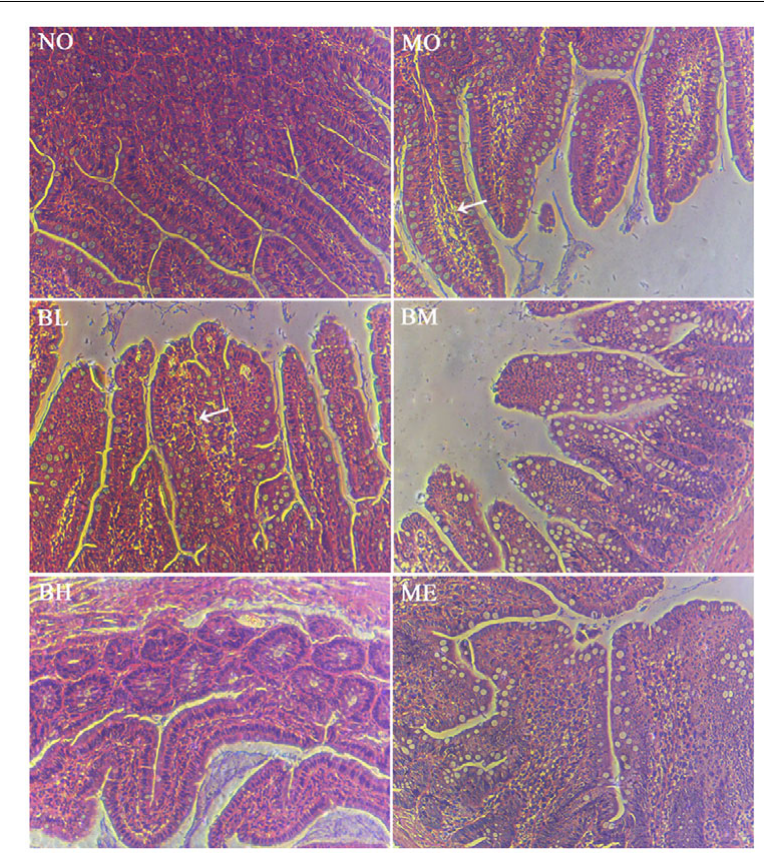

FIGURE 5 | Histology of small bowel with H\&E staining after the intestinal infusion of FITC-dextran. FITC-dextran was different under the intestinal epithelium after intestinal perfusion. White arrowheads suggested more fluorescent dextran underneath the intestinal epithelium was observed. blot. Compared with the rats in NO group, these tight junction proteins expressions were reduced in the intestine of untreated diabetic rats. BBR treatment could partially increase OCLN and ZO-1 expressions but it showed no effects on Claudin-1 levels (Figures 4A-C). After the intestinal perfusion of FITCdextran, BBR could reduce serum dextran concentration, namely the intestinal barrier permeability (Figure 4D). Although HE staining illustrated no significant change in intestinal structure between these groups (Supplementary Figure S1), the amount and distribution of filtered FITCdextran were different in intestinal mucosa and submucosa tissues after intestinal dextran perfusion (Figure 5), which also suggested differences of the intestinal permeability in the rats of various groups. With regard to the ultrastructure of intestinal epithelial cells, intercellular adhesions were damaged, and the adherent junction in diabetic rats was sparse and expanded. These changes were improved after BBR treatment (Figure 6).

\section{BBR Modulates the Expressions of the Molecules Involved in TLR4/MyD88/NF-кB Signaling Pathways in Intestines of Diabetic Rats}

Previous studies confirmed that BBR could protect islet beta cells in DM, and also prevent intestinal inflammation in bowel disease through depressing TLR4 signaling pathways (Zhang et al., 


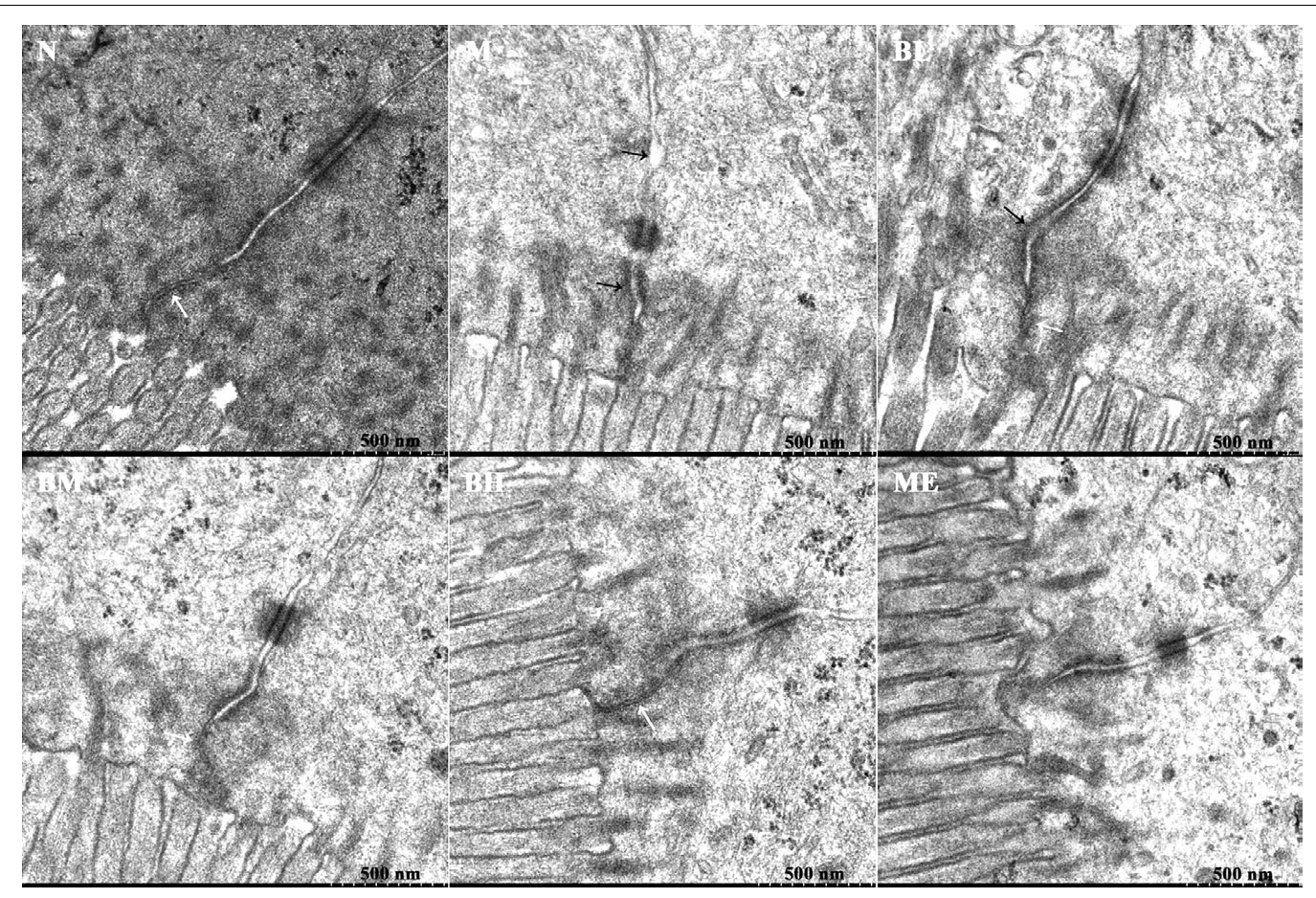

FIGURE 6 | Ultrastructure of intestinal intercellular junction in different groups. The figures revealed the disrupted and expanded adherens junctions of intestinal epithelial cells in $\mathrm{MO}$ and $\mathrm{BL}$ groups, although the desmosome was visible in each group. Black arrowheads indicated damaged cell-cell adhesion and wider gaps between adjacent intestinal epithelial cells. White arrowheads suggested the tight junctions.

2011). To ascertain whether the hypoglycemic and gut-protective effects of BBR were mediated through anti-inflammatory signal transduction pathways, we detected the expressions of TLR4 and downstream MyD88 and $\mathrm{p}-\mathrm{IKK} \beta$ in the intestines, along with p65 NF- $\kappa$ B distributions in epithelial cytoplasm and nucleus. The results revealed $\mathrm{BBR}$, especially at a high dose, reduced intestinal TLR4 and MyD88 protein expressions as well as the phosphorylation of IKK $\beta$ (Figures 7A-C). As a main ligand for TLR4, LPS combined with LPS binding protein (LBP) and CD14 to form the trimer; then the complex binds to the TLR4 and activates inflammatory signal transduction pathways. Data of RT-PCR illustrated intestinal LBP and CD14 mRNA levels decreased in BBR-treated rats (Figures 7D,E). Accordingly, Figure 8 showed p65 NF- $\kappa$ B distributions were different in intestinal epithelial cells among different groups, and BBR exerted a potential ability to blunt $\mathrm{p} 65 \mathrm{NF}-\kappa \mathrm{B}$ translocation to the nuclei.

\section{DISCUSSION}

T2DM rat model was established by HGFD and intravenous injection of $24 \mathrm{mg} / \mathrm{kg} \mathrm{STZ}$ in this study (Duca et al., 2015). Consistent with previous studies, decreased body weights were observed after the STZ treatment (Wang et al., 2016). After BBR delivery with the dose range between 93.75 and $375 \mathrm{mg} / \mathrm{kg}$, the hypoglycemic and hypolipidemic efficacies were shown in a dosedependent manner. Previous researches have shown that the bioavailability of BBR was lower than 5\% (Pirillo and Catapano, 2015), and it seems inexplicable for the valid hypoglycemic effects with such low bioavailability. We then investigated whether BBR exerted beneficial therapeutic impacts on DM through the intestines.

Though human and rodent studies confirmed the proportion changes of immune cells in gut LP of diabetic mice (MonteiroSepulveda et al., 2015), the transplantation of MLN can transmit diabetes to the recipient mice, which highlights the vital pathogenic effects of MLN (Antvorskov et al., 2014; Winer et al., 2016). Therefore we firstly focused on the immune cells population alterations in the MLN of diabetic rats. Consistent with the changes in intestinal LP, we observed reduced Tregs and increased macrophages in diabetic rats, which was inhibited by BBR treatment. Previous studies also found CD4+, CD8+ and DC cells went through transformations in LP (Monteiro-Sepulveda et al., 2015), but no significant differences about aforementioned cells were discovered in the present experiment. It might be explained by different high fat diet or treatment duration, which led to the differentia in intestinal bacteria spectrum and the variant activated immune cells. Based on proinflammatory change of immune cells, we observed that intestinal inflammatory cytokines IL-1 $\beta$, TNF$\alpha$ and MIF-1 increased, and IL-10 and IL- 4 which maintain immune tolerance reduced in diabetic rats in the present study. These intestinal cytokine changes in diabetic rats were consistent with the data in clinical trials; namely, intestinal cytokine's milieu of healthy persons features high levels of 

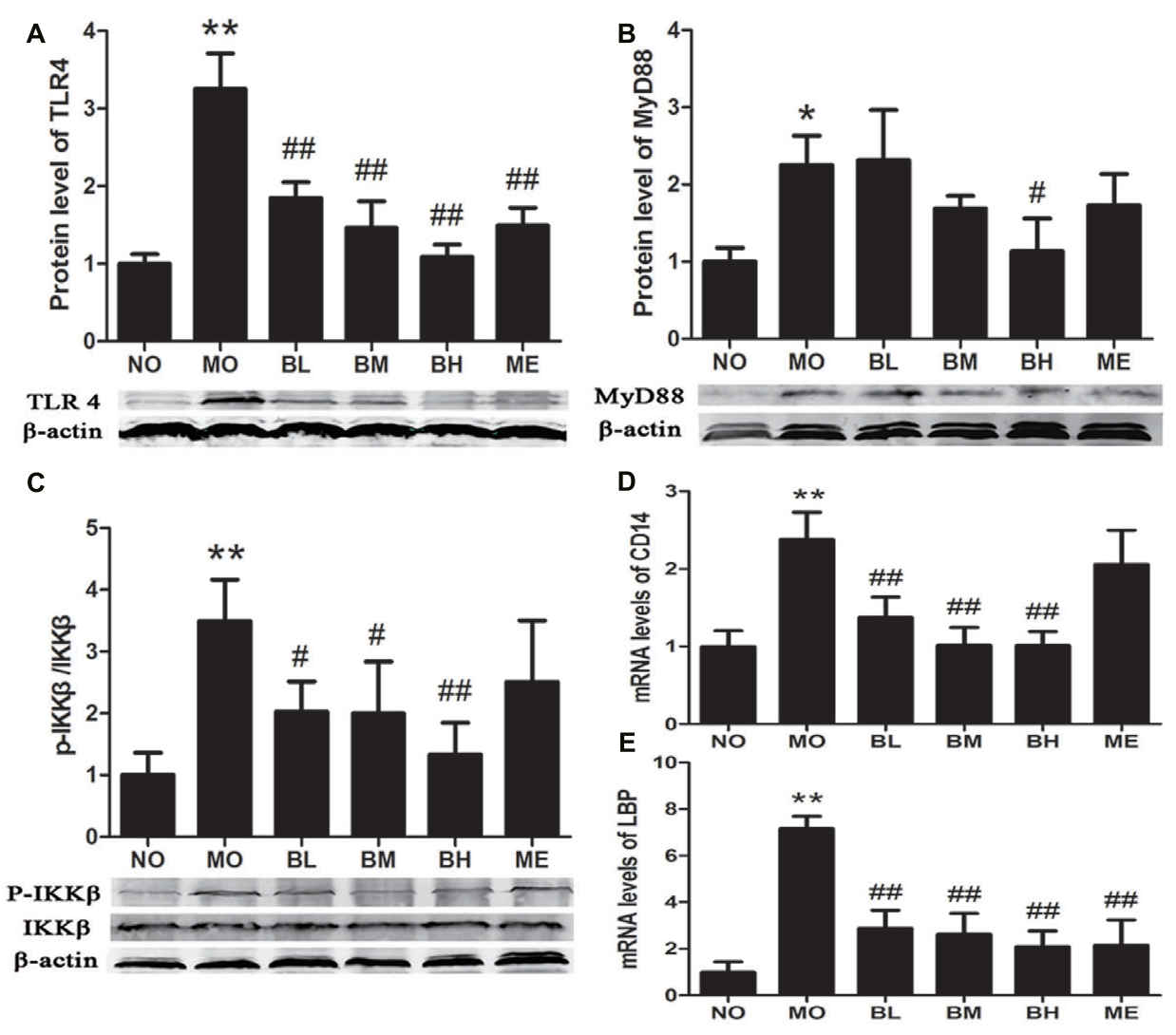

FIGURE 7| The gut-protective effects of BBR are related to TLR4/MyD88/NF $\mathbf{k}$ signaling pathway. (A) TLR4 expressions in intestine ( $n=4)$. (B) MyD88 expressions in intestine $(n=3)$. (C) Fold change of $p-\operatorname{IKK} \beta / \mathrm{IKK} \beta$ ratios in intestine $(n=4)$. (D) The mRNA levels of CD14 $(n=6)$. (E) The mRNA levels of LBP $(n=6)$. The levels of mRNA were expressed relative to the $\beta$-actin. The protein sampling mass was $200 \mu \mathrm{g}$ for TLR4 and MyD88; while $100 \mu \mathrm{g}$ for $\mathrm{p}$-IKK $\beta$ and IKK $\beta$. Data presented as mean \pm SD. ${ }^{*} P<0.05$ vs. NO group, ${ }^{* *} P<0.01$ vs. NO group, ${ }^{\#} P<0.05$ vs. MO group, ${ }^{\# \#} P<0.01$ vs. MO group.

sIgA and IL-10 (Fritz et al., 2012) while that of subjects with metabolic disease features increased proinflammatory cytokines in the intestinal LP and epithelium (Garidou et al., 2015).

How did these pro-inflammatory changes occur in intestinal immune system of diabetic rats? How did they affect the development of DM? HGFD was found to induce decreased expressions and abnormal distributions of intestinal tight junction proteins, and then intestinal permeability was increased (Everard et al., 2013; Wang et al., 2014). Once the mucosal barrier was damaged, luminal LPS, DNA and other bacterial products went through bowels, activated the immune cells with the expressions of CD14 and TLR4 (Delzenne et al., 2015; Plociennikowska et al., 2015; Winer et al., 2016), and caused abnormal secretions of immune cytokine (He et al., 2016). With proinflammatory cytokines binding to responding receptors, insulin signaling pathways were interfered, and abnormal insulin secretion and insulin resistance emerged (Caricilli et al., 2011). Besides, in chronic inflammatory state of DM, proinflammatory cytokines such as IL-1 $\beta$, IL-6 and TNF- $\alpha$ aggravated the impairment in intestinal mucosal barrier (Winer et al., 2016). In the present study, tight junction proteins OCLN, ZO-1 and Claudin-1 expressions were decreased, and serum FITC-dextran was increased after the luminal dextran perfusion in diabetic rats. BBR seemed to protect the intestinal barrier integrity through modulating $\mathrm{ZO}-1$ and OCLN expressions; however, BBR did not influence the Claudin1 level in our study. Claudin-1 is a member of claudin family and an essential component in intestinal mucosal barrier forming. The intestinal proinflammatory immune factor and $\mathrm{NF}-\kappa \mathrm{B}$ could induce the decrease in claudin-1 expression inflammatory bowel disease (Zhou et al., 2009), and BBR might regulate claudin- 4 and claudin-2 expressions other than claudin1 in claudin family (Amasheh et al., 2010; Li et al., 2014). In the present study, we also found that BBR could blunt TLR4 expression and attenuate local metabolic inflammation possibly through suppressing TLR4/MyD88/IKK $\beta$ signaling pathways in intestinal tissue. It might be one of the molecular mechanisms for BBR to protect the intestinal mucosal barrier and inhibit the proinflammatory changes in the treatment of DM.

Additionally, we observed the secretion of GLP-1 and GIP was reduced in diabetic rats, and the concentration of Amylin was increased despite of no significant change of Ghrelin. BBR, especially at a high concentration, prevented the aberrant changes of the hormones, which might be related to 


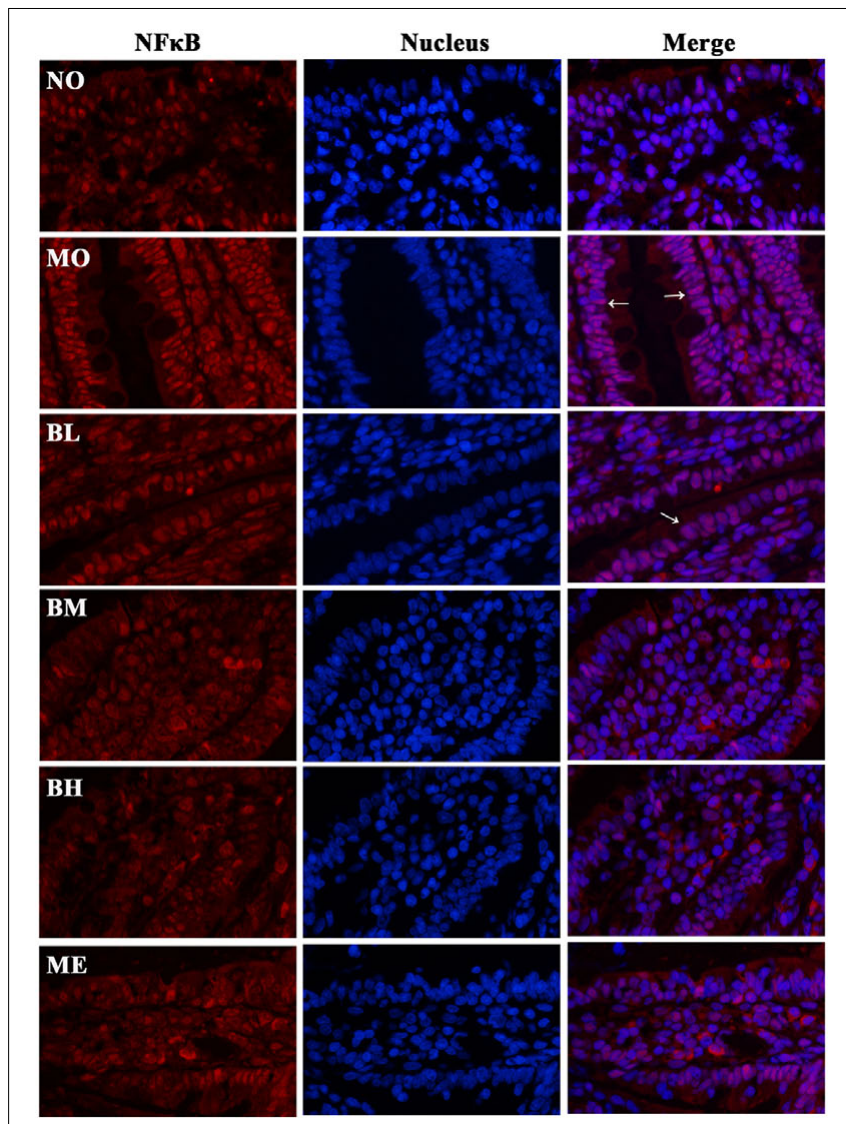

FIGURE 8 | Representative immunofluorescence staining images of p65 NF-кB in small bowels. Transcription factor NF-кB is widely expressed in the intestines, and the distribution in nucleus and cytoplasm is different under various conditions. The results suggested the distributions of p65 $\mathrm{NF}-\kappa \mathrm{B}$ (red) were different in the cytoplasm and nuclei of intestinal epithelium among different groups. Nuclei were stained blue with DAPI. BBR might reduce the translocation of $\mathrm{p} 65 \mathrm{NF}-\mathrm{kB}$ into the nuclei (pink). White arrowheads indicated the distributions of p65 NF-kB in the nuclei of intestinal epithelial cells.

its inhibitory effects on intestinal proinflammatory cells and cytokines. These gastrointestinal hormones GLP-1, GIP and Ghrelin, and pancreatic beta cells-derived Amylin, can modulate glucose metabolism (Westwell-Roper et al., 2014). Intestinal immune molecules could also affect GLP-1 secretion from ileal $\mathrm{L}$ cells, and anti-TNF- $\alpha$ therapy could reverse high fat diet induced reduction of GLP-1 in diabetic mice (Everard et al., 2013; Anhe et al., 2015; Luck et al., 2015; Yusta et al., 2015). Doubtfully, Ghrelin concentrations in the rats of NO group appeared to be higher than those of $\mathrm{BH}$ group despite of no statistically significance. Ghrelin was secreted from gastric $\mathrm{P} / \mathrm{D} 1$ cells and pancreatic $\varepsilon$ cells, which was influenced by many factors such as STZ, HGFD, gut microbiota, bitter taste receptors, and so on (Janssen et al., 2011); STZ could increase its secretion (Muller et al., 2015) while high-fat diet could reduce its secretion (Little and Feinle-Bisset, 2011). Previous study had proved that BBR might regulate the secretion of GLP-1 via intestinal bitter taste receptor in enteric L cells (Yu et al., 2015). Further studies are required to confirm whether BBR could influence bitter taste receptors in ghrelin-secreting cells.

The intestinal improvements in the diabetic rats receiving BBR administration gave rise to question whether intestines were the exclusive hypoglycemic action sites of BBR. When the absorption rate of BBR was enhanced, the hypoglycemic effect was better (Zhaojie et al., 2014; Pirillo and Catapano, 2015). Therefore, BBR may exert its anti-diabetic effect through multiple ways, and the target in the intestine is probably just one of them. Consistent with preceding studies, the positive control metformin could promote the secretion of GLP-1 (McCreight et al., 2016). Although metformin had obvious gastrointestinal side-effect in patients, it exhibited a protective effect on the expression of OCLN. In addition, metformin could reduce TLR4 expressions, increase Tregs and decrease the proportion of macrophages. Such effects might be related to the increased expression of intestinal probiotics as previously reported (Forslund et al., 2015).

Intestinal immune system, endocrine system, mucosal barrier and gut microbiota interact with each other and have crosstalks, but the initial lesion and subsequent reaction are not fully clear. Further elaborate in vivo and ex vivo experiments need to be performed to confirm BBR preliminary acts on one or some of the sites. Taken together, these findings suggested the abnormities of intestinal mucosal barrier, immune system, endocrine and PPRs in diabetic rats. However, BBR could improve the barrier function defect, depress the proinflammatory changes of intestinal immune cells and cytokines, and change aberrant gut-derived hormones which might be pharmacological mechanisms for treating diabetes. However, the interaction among them and the exact anti-diabetic mechanism of BBR need to be further studied.

\section{AUTHOR CONTRIBUTIONS}

FL, HD, and JG designed the study. JG, MH, ZH, KF, QC, JL, DY, and $\mathrm{XZ}$ conducted the experiments. JG, HD, KF, and FL wrote and revised the manuscript. DW, LX, and KW also contributed to the design. All authors approved the final version to be published.

\section{ACKNOWLEDGMENT}

This article is supported by the National Natural Science Foundation of China (No. 81473637, 81373871, 81473448).

\section{SUPPLEMENTARY MATERIAL}

The Supplementary Material for this article can be found online at: http://journal.frontiersin.org/article/10.3389/fphar.2017. 00042/full\#supplementary-material

FIGURE S1 | Histology of small bowel with H\&E staining in each group. No obvious pathological differences were shown in intestinal tissue histology with $\mathrm{H} \& \mathrm{E}$ staining among $\mathrm{NO}, \mathrm{MO}$ and various treatment groups. 


\section{REFERENCES}

Amasheh, M., Fromm, A., Krug, S. M., Amasheh, S., Andres, S., Zeitz, M., et al. (2010). TNFalpha-induced and berberine-antagonized tight junction barrier impairment via tyrosine kinase, Akt and NFkappaB signaling. J. Cell Sci. 123, 4145-4155. doi: 10.1242/jcs.070896

Anhe, F. F., Roy, D., Pilon, G., Dudonne, S., Matamoros, S., Varin, T. V., et al. (2015). A polyphenol-rich cranberry extract protects from diet-induced obesity, insulin resistance and intestinal inflammation in association with increased Akkermansia spp. population in the gut microbiota of mice. Gut 64, 872-883. doi: 10.1136/gutjnl-2014-307142

Antvorskov, J. C., Josefsen, K., Engkilde, K., Funda, D. P., and Buschard, K. (2014). Dietary gluten and the development of type 1 diabetes. Diabetologia 57, 1770-1780. doi: 10.1007/s00125-014-3265-1

Burcelin, R. (2012). Regulation of metabolism: a cross talk between gut microbiota and its human host. Physiology 27, 300-307. doi: 10.1152/physiol.00023.2012

Caricilli, A. M., Picardi, P. K., de Abreu, L. L., Ueno, M., Prada, P. O., Ropelle, E. R., et al. (2011). Gut microbiota is a key modulator of insulin resistance in TLR 2 knockout mice. PLoS Biol. 9:e1001212. doi: 10.1371/journal.pbio.1001212

DeFronzo, R. A., Buse, J. B., Kim, T., Burns, C., Skare, S., Baron, A., et al. (2016). Once-daily delayed-release metformin lowers plasma glucose and enhances fasting and postprandial GLP-1 and PYY: results from two randomised trials. Diabetologia 59, 1645-1654. doi: 10.1007/s00125-016-3992-6

Delzenne, N. M., Cani, P. D., Everard, A., Neyrinck, A. M., and Bindels, L. B. (2015). Gut microorganisms as promising targets for the management of type 2 diabetes. Diabetologia 58, 2206-2217. doi: 10.1007/s00125-015-3712-7

Dong, H., Wang, N., Zhao, L., and Lu, F. (2012). Berberine in the treatment of type 2 diabetes mellitus: a systemic review and meta-analysis. Evid Based Complement. Alternat. Med. 2012:591654. doi: 10.1155/2012/591654

Dong, H., Zhao, Y., Zhao, L., and Lu, F. (2013). The effects of berberine on blood lipids: a systemic review and meta-analysis of randomized controlled trials. Planta Med. 79, 437-446. doi: 10.1055/s-0032-1328321

Duca, F. A., Cote, C. D., Rasmussen, B. A., Zadeh-Tahmasebi, M., Rutter, G. A., Filippi, B. M., et al. (2015). Metformin activates a duodenal Ampk-dependent pathway to lower hepatic glucose production in rats. Nat. Med. 21, 506-511. doi: $10.1038 / \mathrm{nm} .3787$

Everard, A., Belzer, C., Geurts, L., Ouwerkerk, J. P., Druart, C., Bindels, L. B., et al. (2013). Cross-talk between Akkermansia muciniphila and intestinal epithelium controls diet-induced obesity. Proc. Natl. Acad. Sci. U.S.A. 110, 9066-9071. doi: $10.1073 /$ pnas. 1219451110

Forslund, K., Hildebrand, F., Nielsen, T., Falony, G., Le Chatelier, E., Sunagawa, S., et al. (2015). Disentangling type 2 diabetes and metformin treatment signatures in the human gut microbiota. Nature 528, 262-266. doi: 10.1038/nature 15766

Fritz, J. H., Rojas, O. L., Simard, N., McCarthy, D. D., Hapfelmeier, S., Rubino, S., et al. (2012). Acquisition of a multifunctional IgA+ plasma cell phenotype in the gut. Nature 481, 199-203. doi: 10.1038/nature10698

Garidou, L., Pomie, C., Klopp, P., Waget, A., Charpentier, J., Aloulou, M., et al. (2015). The gut microbiota regulates intestinal CD4 T cells expressing RORgammat and controls metabolic disease. Cell Metab. 22, 100-112. doi: 10.1016/j.cmet.2015.06.001

Graham, S., Courtois, P., Malaisse, W. J., Rozing, J., Scott, F. W., and Mowat, A. M. (2004). Enteropathy precedes type 1 diabetes in the BB rat. Gut 53, 1437-1444. doi: 10.1136/gut.2004.042481

He, Y., Liu, S., Kling, D. E., Leone, S., Lawlor, N. T., Huang, Y., et al. (2016). The human milk oligosaccharide 2'-fucosyllactose modulates CD14 expression in human enterocytes, thereby attenuating LPS-induced inflammation. Gut. 65, 33-46. doi: 10.1136/gutjnl-2014-307544

Janssen, S., Laermans, J., Verhulst, P. J., Thijs, T., Tack, J., and Depoortere, I. (2011). Bitter taste receptors and alpha-gustducin regulate the secretion of ghrelin with functional effects on food intake and gastric emptying. Proc. Natl. Acad. Sci. U.S.A. 108, 2094-2099. doi: 10.1073/pnas.1011508108

Lancet (2016a). Worldwide trends in diabetes since 1980: a pooled analysis of 751 population-based studies with 4.4 million participants. Lancet 387, 1513-1530. doi: 10.1016/S0140-6736(16)00618-8

Lancet (2016b). Beat diabetes: an urgent call for global action. Lancet 387:1483. doi: 10.1016/S0140-6736(16)30185-4
Leng, S. H., Lu, F. E., and Xu, L. J. (2004). Therapeutic effects of berberine in impaired glucose tolerance rats and its influence on insulin secretion. Acta Pharmacol. Sin. 25, 496-502.

Li, G. X., Wang, X. M., Jiang, T., Gong, J. F., Niu, L. Y., and Li, N. (2014). Berberine prevents damage to the intestinal mucosal barrier during early phase of sepsis in rat through mechanisms independent of the NOD-like receptors signaling pathway. Eur. J. Pharmacol. 730, 1-7. doi: 10.1016/j.ejphar.2014.1002.1006

Little, T. J., and Feinle-Bisset, C. (2011). Effects of dietary fat on appetite and energy intake in health and obesity-oral and gastrointestinal sensory contributions. Physiol. Behav. 104, 613-620. doi: 10.1016/j.physbeh.2011.04.038

Luck, H., Tsai, S., Chung, J., Clemente-Casares, X., Ghazarian, M., Revelo, X. S., et al. (2015). Regulation of obesity-related insulin resistance with gut anti-inflammatory agents. Cell Metab. 21, 527-542. doi: 10.1016/j.cmet.2015. 03.001

Mardinoglu, A., Boren, J., and Smith, U. (2016). Confounding effects of metformin on the human gut microbiome in type 2 diabetes. Cell Metab. 23, 10-12. doi: 10.1016/j.cmet.2015.1012.1012

McCreight, L. J., Bailey, C. J., and Pearson, E. R. (2016). Metformin and the gastrointestinal tract. Diabetologia 59, 426-435. doi: 10.1007/s00125-0153844-9

Monteiro-Sepulveda, M., Touch, S., Mendes-Sa, C., Andre, S., Poitou, C., Allatif, O., et al. (2015). Jejunal T cell inflammation in human obesity correlates with decreased enterocyte insulin signaling. Cell Metab. 22, 113-124. doi: 10. 1016/j.cmet.2015.05.020

Muller, T. D., Nogueiras, R., Andermann, M. L., Andrews, Z. B., Anker, S. D., Argente, J., et al. (2015). Ghrelin. Mol Metab. 4, 437-460. doi: 10.1016/j.molmet. 2015.03.005

Pirillo, A., and Catapano, A. L. (2015). Berberine, a plant alkaloid with lipidand glucose-lowering properties: from in vitro evidence to clinical studies. Atherosclerosis 243, 449-461. doi: 10.1016/j.atherosclerosis.2015.09.032

Plociennikowska, A., Hromada-Judycka, A., Borzecka, K., and Kwiatkowska, K. (2015). Cooperation of TLR4 and raft proteins in LPS-induced proinflammatory signaling. Cell Mol. Life Sci. 72, 557-581. doi: 10.1007/s00018014-1762-5

Shin, N. R., Lee, J. C., Lee, H. Y., Kim, M. S., Whon, T. W., Lee, M. S., et al. (2014). An increase in the Akkermansia spp. population induced by metformin treatment improves glucose homeostasis in diet-induced obese mice. Gut 63, 727-735. doi: 10.1136/gutjnl-2012-303839

Turner, N., Li, J. Y., Gosby, A., To, S. W., Cheng, Z., Miyoshi, H., et al. (2008). Berberine and its more biologically available derivative, dihydroberberine, inhibit mitochondrial respiratory complex I: a mechanism for the action of berberine to activate AMP-activated protein kinase and improve insulin action. Diabetes Metab. Res. Rev. 57, 1414-1418. doi: 10.2337/db07-1552

Wang, J., Teng, L., Liu, Y., Hu, W., Chen, W., Hu, X., et al. (2016). Studies on the antidiabetic and antinephritic activities of paecilomyces hepiali water extract in diet-streptozotocin-induced diabetic sprague dawley rats. J. Diabetes Res. 2016, 4368380. doi: 10.1155/2016/4368380

Wang, X., Ota, N., Manzanillo, P., Kates, L., Zavala-Solorio, J., Eidenschenk, C., et al. (2014). Interleukin-22 alleviates metabolic disorders and restores mucosal immunity in diabetes. Nature 514, 237-241. doi: 10.1038/nature13564

Westwell-Roper, C. Y., Ehses, J. A., and Verchere, C. B. (2014). Resident macrophages mediate islet amyloid polypeptide-induced islet IL-1beta production and beta-cell dysfunction. Diabetes Metab. Res. Rev. 63, 1698-1711. doi: $10.2337 / \mathrm{db} 13-0863$

Winer, D. A., Luck, H., Tsai, S., and Winer, S. (2016). The intestinal immune system in obesity and insulin resistance. Cell Metab. 23, 413-426. doi: 10.1016/j.cmet. 2016.01.003

Xu, Y., Wang, L., He, J., Bi, Y., Li, M., Wang, T., et al. (2013). Prevalence and control of diabetes in Chinese adults. JAMA 310, 948-959. doi: 10.1001/jama. 2013.168118

Yu, Y., Hao, G., Zhang, Q., Hua, W., Wang, M., Zhou, W., et al. (2015). Berberine induces GLP-1 secretion through activation of bitter taste receptor pathways. Biochem. Pharmacol. 97, 173-177. doi: 10.1016/j.bcp.2015.07.012

Yusta, B., Baggio, L. L., Koehler, J., Holland, D., Cao, X., Pinnell, L. J., et al. (2015). GLP-1R agonists modulate enteric immune responses through the intestinal intraepithelial lymphocyte GLP-1R. Diabetes Metab. Res. Rev. 64, 2537-2549. doi: $10.2337 / \mathrm{db} 14-1577$ 
Zhang, Q., Piao, X. L., Piao, X. S., Lu, T., Wang, D., and Kim, S. W. (2011). Preventive effect of coptis chinensis and berberine on intestinal injury in rats challenged with lipopolysaccharides. Food Chem. Toxicol. 49, 61-69. doi: 10. 1016/j.fct.2010.09.032

Zhao, H. L., Sui, Y., Qiao, C. F., Yip, K. Y., Leung, R. K., Tsui, S. K., et al. (2012). Sustained antidiabetic effects of a berberine-containing Chinese herbal medicine through regulation of hepatic gene expression. Diabetes Metab. Res. Rev. 61, 933-943. doi: 10.2337/db11-1164

Zhaojie, M., Ming, Z., Shengnan, W., Xiaojia, B., Hatch, G. M., Jingkai, G., et al. (2014). Amorphous solid dispersion of berberine with absorption enhancer demonstrates a remarkable hypoglycemic effect via improving its bioavailability. Int. J. Pharm. 467, 50-59. doi: 10.1016/j.ijpharm.2014.1003.1017

Zhou, W., Cao, Q., Peng, Y., Zhang, Q. J., Castrillon, D. H., DePinho, R. A., et al. (2009). FoxO4 inhibits NF-kappaB and protects mice against colonic injury and inflammation. Gastroenterology 137, 1403-1414. doi: 10.1053/j.gastro.2009.06 049

Conflict of Interest Statement: The authors declare that the research was conducted in the absence of any commercial or financial relationships that could be construed as a potential conflict of interest.

Copyright (c) 2017 Gong, Hu, Huang, Fang, Wang, Chen, Li, Yang, Zou, Xu, Wang, Dong and Lu. This is an open-access article distributed under the terms of the Creative Commons Attribution License (CC BY). The use, distribution or reproduction in other forums is permitted, provided the original author(s) or licensor are credited and that the original publication in this journal is cited, in accordance with accepted academic practice. No use, distribution or reproduction is permitted which does not comply with these terms. 